LETTER TO THE EDITORS

\section{Chikungunya-induced manic episode in a patient with no psychiatric history: a case report}

Braz J Psychiatry. 2020 Nov-Dec;42(6):687

doi:10.1590/1516-4446-2020-0892

\section{(cc) BY-NC}

Chikungunya is a vector-borne zoonosis caused by the chikungunya virus (CHIKV) and transmitted by mosquitoes of the genus Aedes. An outbreak of the disease occurred in Brazil from 2015 to 2017, causing multiple deaths and severe consequences for survivors. Little is known of the potential psychiatric sequelae of CHIKV infection, but studies have demonstrated that depressive episodes, anxiety, and somatoform disorders may occur during infection and may strongly affect patient quality of life. ${ }^{1,2}$ A significant elevation of pro-inflammatory cytokine levels (interleukin [IL]-6, IL-1 $\beta$, IL-2, and tumor necrosis factor $[T N F]-\alpha)$, both circulating and within the central nervous system (CNS), has been identified in patients with bipolar disorder, especially during depressive and manic episodes. Excessive cytokine production in the CNS may lead to apoptosis of neurons in vital neural pathways, including those involved in mood. ${ }^{3}$

We report the case of a 53-year-old homemaker with no personal or familial psychiatric history who presented with sudden onset of fever, skin rash, and right ankle edema. Acute CHIKV infection was diagnosed by serologic testing (IgM) in the emergency department (ED). Inflammatory markers, including C-reactive protein and erythrocyte sedimentation rate (ESR), were elevated. After 5 days, she developed manic symptoms, including decreased need for sleep, logorrhea, psychomotor agitation, excessive energy, irritation, racing thoughts, and grandiosity. She returned to the ED with a chief complaint of sudden change of behavior, agitation, and disinhibition. General blood tests were negative, and computed tomography (CT) of the head was within normal limits. She denied use of any drugs or medicines other than those prescribed during her previous ED visit, and a toxicology panel was negative. There were no hallucinations, delusions, or depressive symptoms.

Thirty days after onset of mania, she was admitted to our outpatient psychiatric clinic and olanzapine $10 \mathrm{mg} /$ day was initiated. Manic symptoms persisted for 45 days after onset of CHIKV symptoms. A comprehensive laboratory workup, which included antinuclear factor, anti-SSA, antiSSB, rheumatoid factor, serologies for HIV, hepatitis $B$ and $\mathrm{C}$ virus, toxoplasmosis, cytomegalovirus, and venereal disease research laboratory (VDRL), was negative. IgG antibodies to CHIKV were detected. Brain magnetic resonance imaging (MRI) showed no relevant alterations. The patient continues to receive follow-up at our service, and has been in remission for 2 years.

To our knowledge, there is no report in the scientific literature of a manic episode induced by chikungunya in a patient with no previous psychiatric history. Like bipolar disorder, CHIKV infection leads to significant cytokine overproduction and CNS inflammation. Both Th1 and Th2 immune responses are activated during the infection, which may trigger mood episodes. ${ }^{4} \mathrm{IL}-6$ has been proposed as a key factor in the pathogenesis of CNS immune response to $\mathrm{CHIKV}$, and is known to be elevated in patients with depressive and manic episodes, indicating a possible link between this infection and mood disorders. ${ }^{5}$ Therefore, we hypothesize that the inflammatory response generated by the viral infection may have triggered a manic episode in the patient reported herein. Considering the magnitude of the chikungunya epidemic and its consequences to public health, describing and presenting instances of CHIKV-induced mania may be of pivotal importance for identification and treatment of future cases.

Douglas S. Soares, ${ }^{1}$ iD Leila Y. Fortaleza, ${ }^{1}$
Matias C. Melo ${ }^{1,2}$ iD
${ }^{1}$ Departamento de Psiquiatria, Hospital de Saúde Mental Professor
Frota Pinto, Fortaleza, CE, Brazil. ${ }^{2}$ Centro de Ciências da Saúde,
Universidade de Fortaleza, Fortaleza, CE, Brazil.

Submitted Feb 07 2020, accepted Apr 02 2020, Epub Jun 012020.

\section{Disclosure}

The authors report no conflicts of interest.

How to cite this article: Soares DS, Fortaleza LY, Melo MC. Chikungunya-induced manic episode in a patient with no psychiatric history: a case report. Braz J Psychiatry. 2020;42:687. http://dx.doi.org/10.1590/1516-4446-20200892

\section{References}

1 Figueiredo T, dias da Costa M, Segenreich D. Manic episode after a chikungunya virus infection in a bipolar patient previously stabilized with valproic acid. J Clin Psychopharmacol. 2018;38:395-7.

2 Bhatia MS, Gautam P, Jhanjee A. Psychiatric morbidity in patients with chikungunya fever: first report from India. J Clin Diagn Res. 2015;9:VC01-VC03.

3 Rosenblat JD, McIntyre RS. Bipolar disorder and inflammation. Psychiatr Clin North Am. 2016;39:125-37.

4 Venugopalan A, Ghorpade RP, Chopra A. Cytokines in acute chikungunya. PLoS One. 2014;9:e111305.

5 Rodríguez-Morales AJ, Hernández-Moncada ÁM, Hoyos-Guapacha KL, Vargas-Zapata SL, Sánches-Zapata JF, Mejía-Bernal YV, et al. Potential relationships between chikungunya and depression: solving the puzzle with key cytokines. Cytokine. 2018;102:161-2. 